\title{
Optimal switching control design for polynomial systems: an LMI approach
}

\author{
Didier Henrion ${ }^{1,2,3}$, Jamal Daafouz ${ }^{4}$, Mathieu Claeys ${ }^{1}$
}

Draft of October 31, 2018

\begin{abstract}
We propose a new LMI approach to the design of optimal switching sequences for polynomial dynamical systems with state constraints. We formulate the switching design problem as an optimal control problem which is then relaxed to a linear programming (LP) problem in the space of occupation measures. This infinitedimensional LP can be solved numerically and approximately with a hierarchy of convex finite-dimensional LMIs. In contrast with most of the existing work on LMI methods, we have a guarantee of global optimality, in the sense that we obtain an asympotically converging (i.e. with vanishing conservatism) hierarchy of lower bounds on the achievable performance. We also explain how to construct an almost optimal switching sequence.
\end{abstract}

\section{Introduction}

A switched system is a particular class of a hybrid system that consists of a set of dynamical subsystems, one of which is active at any instant of time, and a policy for activating and deactivating the subsystems. One may encounter such dynamical systems in a wide variety of application domains such as automotive industry, power systems, aircraft and traffic control, and more generally the area of embedded systems. Switched systems have been the concern of many researchers and many results are available for stability analysis and control design. They put in evidence the important fact that it is possible to orchestrate the subsystems through an adequate switching strategy in order to impose global stability. Interested readers may refer to the survey papers [10, 20, 29, 22] and the interesting and useful books [21, 30] and the references therein.

In this context, switching plays a major role for stability and performance properties. Indeed, switched systems are generally controlled by switched controllers and the control

\footnotetext{
${ }^{1}$ CNRS; LAAS; 7 avenue du colonel Roche, F-31077 Toulouse; France. henrion@laas.fr

${ }^{2}$ Université de Toulouse; UPS, INSA, INP, ISAE; UT1, UTM, LAAS; F-31077 Toulouse; France

${ }^{3}$ Faculty of Electrical Engineering, Czech Technical University in Prague, Technická 2, CZ-16626 Prague, Czech Republic

${ }^{4}$ Université de Lorraine, CRAN, CNRS, IUF, 2 avenue de la fort de Haye, 54516 Vandœuvre cedex, France. jamal.daafouz@univ-lorraine.fr
} 
signal is intrinsically discontinuous. As far as optimality is concerned, several results are also available in two main different contexts:

- the first category of methods exploits necessary optimality conditions, in the form of Pontryagin's maximum principle (the so-called indirect approaches), or through a large nonlinear discretization of the problem (the so-called direct approaches), see [2, 3, 6, 15, 23, 24, 25, 26, 27, 28, 31] for details. Therefore only local optimality can be guaranteed for general nonlinear problems, even when discretization can be properly controlled;

- the second category collects extensions of the performance indexes $H_{2}$ and $H_{\infty}$ originally developped for linear time invariant systems without switching, and use the flexibility of Lyapunov's approach, see for instance [13, 9] and references therein. Even for linear switched systems, the proposed results are based on nonconvex optimization problems (e.g. bilinear matrix inequality conditions) difficult to solve directly. Sufficient linear matrix inequality (LMI) design conditions may be obtained, but at the price of introducing a conservatism (pessimism) which is hard, if not impossible, to evaluate. Since the computation of this optimal strategy is a difficult task, a suboptimal solution is of interest only when it is proved to be consistent, meaning that it imposes to the switched system a performance not worse than the one produced by each isolated subsystem [14].

Despite the interest of these existing approaches, the optimal control problem is not completely solved for switched systems and new strategies are more than welcome, as computationally viable design techniques are missing. In this paper, we consider the problem of designing optimal switching rules in the case of polynomial switched dynamical systems. Classically, we formulate the optimal control switching problem as an optimal control problem with controls being functions of time valued in $\{0,1\}$, and we relax it into a control problem with controls being functions of time valued in $[0,1]$. In contrast with existing approaches following this relaxation strategy, see e.g. [2, 24], relying on Pontryagin's maximum principle, our aim is to apply the approach of [18], which consists in modeling control and trajectory functions as occupation measures. This allows for a convex linear programming (LP) formulation of the optimal control problem. This infinitedimensional LP can be solved numerically and approximately with a hierarchy of convex finite-dimensional LMIs. On the one hand, our approach follows the optimal control modeling framework. On the other hand, it exploits the flexibility and computational efficiency of the convex LMI framework. In contrast with most of the existing work on LMI methods, we have a guarantee of global optimality, in the sense that we obtain an asympotically converging (i.e. with vanishing conservatism) hierarchy of lower bounds on the achievable performance.

The paper is organized as follows: in Section 2 we state the optimal switching problem to be solved, and we propose an alternative, relaxed formulation allowing chattering of the trajectories. In Section 3 we introduce occupation measures as a device to linearize the optimal control problem into an LP problem in the cone of nonnegative measures. In Section 4 we explain how to solve the resulting infinite-dimensional LP with a converging hierarchy of finite-dimensional LMI problems. As explained in Section 5, an approximate 
optimal switching sequence can be extracted from the solutions of the LMI problem, and this is illustrated with classical examples in Section 6. Finally, the paper ends with a sketch of further research lines.

\section{Optimal switching problem}

Consider the optimal control problem

$$
\begin{aligned}
p^{*}=\text { inf } & \int_{0}^{T} l_{\sigma(t)}(t, x(t)) d t \\
\text { s.t. } & \dot{x}(t)=f_{\sigma(t)}(t, x(t)), \quad \sigma(t) \in\{1,2, \ldots, m\} \\
& x(0) \in X_{0}, \quad x(T) \in X_{T} \\
& x(t) \in X, \quad t \in[0, T]
\end{aligned}
$$

with given polynomial velocity field $f_{\sigma(t)} \in \mathbb{R}[t, x]^{n}$ and given polynomial Lagrangian $l_{\sigma(t)} \in \mathbb{R}[t, x]$ indexed by an integer-valued signal $\sigma:[0, T] \rightarrow\{1,2, \ldots, m\}$. System state $x(t)$ belongs to a given compact semialgebraic set $X \subset \mathbb{R}^{n}$ for all $t \in[0, T]$ and the initial state $x(0)$ resp. final state $x(T)$ are constrained to a given compact semialgebraic set $X_{0} \subset X$ resp. $X_{T} \subset X$. In problem (1) the infimum is w.r.t. sequence $\sigma$ and terminal time $T$.

In this paper, for the sake of simplicity, we assume that the terminal time $T$ is finite, that is, we do not consider the asymptotic behavior. Typically, if a solution to problem (1) is expected for a very large or infinite terminal time, we must reformulate the problem by relaxing the state constraints.

Optimal control problem (1) can then be equivalently written as

$$
\begin{aligned}
p^{*}=\inf _{u} & \int_{0}^{T} \sum_{k=1}^{m} l_{k}(t, x(t)) u_{k}(t) d t \\
\text { s.t. } & d x(t)=\sum_{k=1}^{m} f_{k}(t, x(t)) u_{k}(t) d t \\
& x(0) \in X_{0}, \quad x(T) \in X_{T} \\
& x(t) \in X, \quad u(t) \in U, \quad t \in[0, T]
\end{aligned}
$$

where the infimum is with respect to a time-varying vector $u(t)$ which belongs for all $t \in[0, T]$ to the (nonconvex) discrete set

$$
U:=\{(1,0, \ldots, 0),(0,1, \ldots, 0), \ldots,(0,0, \ldots, 1)\} \subset \mathbb{R}^{m} .
$$

In general the infimum in problem (2) is not attained (see our numerical examples later on) and the problem is relaxed to

$$
\begin{aligned}
p_{R}^{*}=\inf & \int_{0}^{T} \sum_{k=1}^{m} l_{k}(t, x(t)) u_{k}(t) d t \\
\text { s.t. } \quad & d x(t)=\sum_{k=1}^{m} f_{k}(t, x(t)) u_{k}(t) d t \\
& x(0) \in X_{0}, \quad x(T) \in X_{T} \\
& x(t) \in X, \quad u(t) \in \operatorname{conv} U, \quad t \in[0, T]
\end{aligned}
$$

where the minimization is now with respect to a time-varying vector $u(t)$ which belongs for all $t \in[0, T]$ to the (convex) simplex

$$
\operatorname{conv} U=\left\{u \in \mathbb{R}^{m}: \sum_{k=1}^{m} u_{k}=1, \quad u_{k} \geq 0, \quad k=1, \ldots, m\right\} .
$$


In [2], problem (3) is called the embedding of problem (1), and it is proved that the set of trajectories of problem (1) is dense (w.r.t. the uniform norm in the space of continuous functions of time) in the set of trajectories of embedded problem (3). Note however that these authors consider the more general problem of switching design in the presence of additional bounded controls in each individual dynamics. To cope with chattering effects due to the simultaneous presence of controls and (initial and terminal) state constraints, they have to introduce a further relaxation of the embedded control problem. In this paper, we do not have controls in the dynamics, and the only design parameter is the switching sequence.

An equivalent way of writing the dynamics in problem (3) is via a differential inclusion

$$
\dot{x}(t) \in \operatorname{conv}\left\{f_{1}(t, x(t)), \ldots, f_{m}(t, x(t))\right\} .
$$

By this it is meant that at time $t$, the state velocity $\dot{x}(t)$ can be any convex combination of the vector fields $f_{k}(t, x(t)), k=1, \ldots, m$, see e.g. [4, Section 3.1] for a tutorial introduction. For this reason, problem (3) is also sometimes called the convexification of problem (1).

Since problem (3) is a relaxation of problem (1), it holds $p_{R}^{*} \leq p^{*}$. For most of the physically relevant problems, and especially when the state constraints in problem (1) are not overly stringent, it actually holds that $p_{R}^{*}=p^{*}$. For a discussion about the cases for which $p_{R}^{*}<p^{*}$, please refer to [16, Appendix C] and references therein.

\section{Occupation measures}

Given an initial condition $x_{0} \in X_{0}$ and an admissible control $u(t)$, denote by $x\left(t \mid x_{0}, u\right)$, $t \in[0, T]$, the corresponding admissible trajectory, an absolutely continuous function of time with values in $X$. Define the occupation measure

$$
\mu\left(A \times B \mid x_{0}, u\right):=\int_{0}^{T} I_{A \times B}\left(t, x\left(t \mid x_{0}, u\right)\right) d t
$$

for all subsets $A \times B$ in the Borel $\sigma$-algebra of subsets of $[0, T] \times X$, where $I_{A}(x)$ is the indicator function of set $A$, equal to one if $x \in A$, and zero otherwise. We write $x\left(t \mid x_{0}, u\right)$ resp. $\mu\left(d t, d x \mid x_{0}, u\right)$ to emphasize the dependence of $x$ resp. $\mu$ on initial condition $x_{0}$ and control $u$, but for conciseness we also use the notation $x(t)$ resp. $\mu(d t, d x)$. The occupation measure can be disintegrated into

$$
\mu(A \times B)=\int_{A} \xi(B \mid t) \omega(d t)
$$

where $\xi(d x \mid t)$ is the distribution of $x \in \mathbb{R}^{n}$, conditional on $t$, and $\omega(d t)$ is the marginal w.r.t. time $t$, which models the control action as a measure on $[0, T]$. The conditional $\xi$ is a stochastic kernel, in the sense that for all $t \in[0, T], \xi(. \mid t)$ is a probability measure on $X$, and for every $B$ in the Borel $\sigma$-algebra of subsets of $X, \xi(B \mid$.$) is a Borel measurable$ function on $[0, T]$. An equivalent definition is $\xi(B \mid t)=I_{B}(x(t))=\delta_{x(t)}(B)$ where $\delta$ is the Dirac measure. The occupation measure encodes the system trajectory, and the value 
$\int_{0}^{T} \mu(d t, B)=\mu([0, T] \times B)$ is equal to the total time spent by the trajectory in set $B \subset X$. Note also that time integration of any smooth test function $v:[0, T] \times X \rightarrow \mathbb{R}$ along a trajectory becomes a time and space integration against $\mu$, i.e.

$$
\int_{0}^{T} v(t, x(t)) d t=\int_{0}^{T} \int_{X} v(t, x) \mu(d t, d x)=\int v \mu .
$$

In optimal control problem (3), we associate an occupation measure

$$
\mu_{k}(d t, d x)=\xi_{k}(d x \mid t) \omega_{k}(t)
$$

for each system mode $k=1, \ldots, m$, so that globally

$$
\sum_{k=1}^{m} \mu_{k}=\mu
$$

is the occupation measure of a system trajectory subject to switching. The marginal $\omega_{k}$ is the control, modeled as a measure which is absolutely continuous w.r.t. the Lebesgue measure, i.e. such that

$$
\int_{X} \mu_{k}(d t, d x)=\omega_{k}(d t)=u_{k}(t) d t
$$

for some measurable control function $u_{k}(t), k=1, \ldots, m$. The system dynamics in problem (3) can then be expressed as

$$
d x(t)=\sum_{k=1}^{m} f_{k}(x(t)) \omega_{k}(d t)
$$

where the controls are now measures $\omega_{k}$. To enforce that $u(t) \in$ conv $U$ for almost all times $t \in[0, T]$, we add the constraint

$$
\sum_{k=1}^{m} \omega_{k}(d t)=I_{[0, T]}(t) d t
$$

where the right hand side is the Lebesgue measure, or uniform measure on $[0, T]$.

Given a smooth test function $v:[0, T] \times X \rightarrow \mathbb{R}$ and an admissible trajectory $x(t)$ with occupation measure $\mu(d t, d x)$, it holds

$$
\begin{aligned}
& \int_{0}^{T} d v(t, x(t))=v(T, x(T))-v(0, x(0)) \\
& =\int_{0}^{T}\left(\frac{\partial v}{\partial t}(t, x(t))+\sum_{k} \operatorname{grad} v(t, x(t)) f_{k}(t, x(t)) u_{k}(t)\right) d t \\
& =\int_{0}^{T} \int_{X}\left(\frac{\partial v}{\partial t}(t, x) \mu(d t, d x)+\right. \\
& \left.\quad \sum_{k} \operatorname{grad} v(t, x) f_{k}(t, x) \mu_{k}(d t, d x)\right) \\
& =\sum_{k} \int \frac{\partial v}{\partial t} \mu_{k}+\operatorname{grad} v f_{k} \mu_{k} .
\end{aligned}
$$

Now, consider that the initial state is not a single vector $x_{0}$ but a random vector whose distribution is ruled by a probability measure $\mu_{0}$, so that at time $t$ the state $x(t)$ is also modeled by a probability measure $\mu_{t}():.=\xi(. \mid t)$, not necessarily equal to $\delta_{x(t)}$. The interpretation is that $\mu_{t}(B)$ is the probability that the state $x(t)$ belongs to a set $B \subset$ 
$X$. Optimal control problem (3) can then be formulated as a linear programming (LP) problem:

$$
\begin{aligned}
& p_{M}^{*}=\inf \sum_{k} \int l_{k} \mu_{k} \text { s.t. } \\
& \int v \mu_{T}-\int v \mu_{0}=\sum_{k} \int \frac{\partial v}{\partial t} \mu_{k}+\operatorname{grad} v f_{k} \mu_{k} \\
& \sum_{k} \int w \mu_{k}=\int w \\
& \forall v \in C^{1}([0, T] \times X), \quad \forall w \in C^{1}([0, T])
\end{aligned}
$$

where the infimum is w.r.t. measures $\mu_{0} \in M_{+}\left(X_{0}\right), \mu_{T} \in M_{+}\left(X_{T}\right), \mu_{k} \in M_{+}([0, T] \times X)$, $k=1, \ldots, m$ with $M_{+}(A)$ denoting the cone of finite nonnegative measures supported on $A$, identified as the dual of the cone of nonnegative continuous functions supported on $A$.

It follows readily that $p^{*} \geq p_{R}^{*} \geq p_{M}^{*}$, and under some additional assumptions it should be possible to prove that $p_{R}^{*}=p_{M}^{*}$ and that the marginal densities $u_{k}$ extracted from solutions $\mu_{k}$ of problem (7) are optimal for problem (2) and hence problem (1). We leave the rigorous statement and its proof for an extended version of this paper.

Note that the use of relaxations and LP formulations of optimal control problems (on ordinary differential equations and partial differential equations) is classical, and can be traced back to the work by L. C. Young, Filippov, and then Warga and Gamkrelidze, amongst many others. For more details and a historical survey, see e.g. [12, Part III].

\section{Solving the LP on measures}

To summarize, we have formulated our relaxed optimal switching control problem (3) as the convex LP (7) in the space of measures. This can be seen as an extension of the approach of [18] which was originally designed for classical optimal control problems. Alternatively, this can also be understood as an application of the approach of [7] where the control measures are restricted to be absolutely continuous w.r.t. time. Indeed, absolute continuity of the control measures is enforced by relation (6). The infinite-dimensional LP on measures (7) can be solved approximately by a hierarchy of finite-dimensional linear matrix inequality (LMI) problems, see [18, 7, 16] for details (not reproduced here). The main idea behind the hierarchy is to manipulate each measure via its moments truncated to degree $2 d$, where $d$ is a relaxation order, and to use necessary LMI conditions for a vector to contain moments of a measure. The hierarchy then consists of LMI problems of increasing sizes, and it provides a sequence of lower bounds $p_{d}^{*} \leq p^{*}$ which is monotonically increasing, i.e. $p_{d}^{*} \leq p_{d+1}^{*}$ and asymptotically converging, i.e. $\lim _{d \rightarrow \infty} p_{d}^{*}=p^{*}$.

The number of variables $N_{d}$ at the LMI relaxation of order $d$ grows linearly in $m$ (the number of modes), and polynomially in $d$, but the exponent is a linear function of $n$ (the number of states). In practice, given the current state-of-the-art in general-purpose LMI solvers and personal computers, we can expect an LMI problem to be solved in a matter of a few minutes provided the problem is reasonably well-conditioned and $N_{d} \leq 5000$. 


\section{Optimal switching sequence}

Let

$$
y_{k, \alpha}:=\int_{0}^{T} \int_{X} t^{\alpha} \mu_{k}(d t, d x)=\int_{0}^{T} t^{\alpha} \omega_{k}(d t), \quad \alpha=0,1, \ldots
$$

denote the moments of measure $\omega_{k}, k=1, \ldots, m+1$. Solving the LMI relaxation of order $d$ yields real numbers $\left\{y_{k, \alpha}^{d}\right\}_{\alpha=0,1, \ldots, 2 d}$ which are approximations to $y_{k, \alpha}$.

In particular, the zero order moment of each measure $\mu_{k}$ is an approximation of its mass, and hence of the time $t_{k}=\int \mu_{k} \in[0, T]$ spent by an optimal switching sequence on mode $k$. At each LMI relaxation $d$, it holds $\sum_{k=1}^{m+1} y_{k, 0}^{d}=1$ for all $d$, and $\lim _{d \rightarrow \infty} y_{k, 0}^{d}=t_{k}$, so that in practice, it is expected that good approximations of $t_{k}$ are obtained at relatively small relaxation orders.

The (approximate) higher order moments $\left\{y_{k, \alpha}^{d}\right\}_{\alpha=1, \ldots, 2 d}$ allow to recover (approximately) the densities $u_{k}(t)$ of each measure $\omega_{k}(d t)$, for $k=1, \ldots, m+1$. The problem of recovering a density from its moments is a well-studied inverse problem of numerical analysis. Since we expect in many cases the density to be piecewise constant, with possible discontinuities here corresponding to commutations between system modes, we propose the following strategy.

Let us assume that we have the moments

$$
y_{\alpha}:=\int_{0}^{T} t^{\alpha} \omega(d t)
$$

of a (nonnegative) measure with piecewise constant density

$$
\omega(d t)=u(t) d t:=\sum_{k=1}^{N} u_{k} I_{\left[t_{k-1}, t_{k}\right]}(t) d t
$$

such that the boundary values are zero, i.e. $u_{0}=0$ and $u_{N+1}=0$. The Radon-Nikodym derivative of this measure reads

$$
u^{\prime}(d t)=\sum_{k=1}^{N}\left(u_{k+1}-u_{k}\right) \delta_{t_{k}}(d t)
$$

where $\delta_{t_{k}}$ denotes the Dirac measure at $t=t_{k}$. Let

$$
y_{\alpha}^{\prime}:=\int_{0}^{T} t^{\alpha} u^{\prime}(d t)=\sum_{k=1}^{N}\left(u_{k+1}-u_{k}\right) t_{k}^{\alpha}
$$

denote the moments of the (signed) derivative measure. By integration by parts it holds

$$
y_{\alpha}^{\prime}=-\alpha y_{\alpha-1}, \quad \alpha=0,1,2, \ldots
$$

which shows that the moments of $u^{\prime}$ can be obtained readily from the moments of $u$. Since $u^{\prime}$ is a sum of $N$ Dirac measures, the moment matrix of $u^{\prime}$ is a (signed) sum of $N$ rank-one moment matrices, and the atoms $t_{k}$ as well as the weights $u_{k+1}-u_{k}, k=1, \ldots, N$ can 
be obtained readily from an eigenvalue decomposition of the moment matrix as explained e.g. in [19, Section 4.3].

More generally, the reader interested in numerical methods for reconstructing a measure from the knowledge of its moments is referred to [17] and references therein, as well as to the recent works [11, 1, 5] which deal with the problem of reconstructing a piecewisesmooth function from its Fourier coefficients. To make the connection between moments and Fourier coefficients, let us just mention that the moments $y_{\alpha}=\int t^{\alpha} u(t) d t$ of a smooth density $u(t)$ are (up to scaling) the Taylor coefficients of the Fourier transform $\hat{u}(s):=$ $\int e^{-2 \pi i s t} u(t) d t=\sum_{\alpha} \frac{(-2 \pi i)^{\alpha}}{\alpha !} y_{\alpha} s^{\alpha}$. If the $y_{\alpha}$ are given, then $\hat{u}(s)$ is given by its Taylor series, and the density $u(t)$ is recovered with the inverse Fourier transform $u(t)=\int e^{2 \pi i s t} \hat{u}(s) d s$. Numerically, an approximate density can be obtained by applying the inverse fast Fourier transform to the (suitably scaled) sequence $\left\{y_{\alpha}^{d}\right\}_{\alpha=0,1, \ldots, 2 d}$ of moments.

\section{Examples}

\subsection{First example}

Consider the scalar $(n=1)$ optimal control problem $(1)$ :

$$
\begin{aligned}
p^{*}=\inf & \int_{0}^{1} x^{2}(t) d t \\
\text { s.t. } \quad & \dot{x}(t)=a_{\sigma(t)} x(t) \\
& x(0)=\frac{1}{2}, \quad x(1) \in[-1,1] \\
& x(t) \in[-1,1], \quad \forall t \in[0,1]
\end{aligned}
$$

where the infimum is w.r.t. to a switching sequence $\sigma:[0,1] \mapsto\{1,2\}$ and

$$
a_{1}:=-1, \quad a_{2}:=1 \text {. }
$$

In Table 1 we report the lower bounds $p_{d}^{*}$ on the optimal value $p^{*}$ obtained by solving LMI relaxations of increasing orders $d$, rounded to 5 significant digits. We also indicate the number of variables (i.e. total number of moments) of each LMI problem, as well as the zeroth order moment of each occupation measure (recall that these are approximations of the time spent on each mode). We observe that the values of the lower bounds and the masses stabilize quickly.

In this simple case, it is easy to obtain analytically the optimal switching sequence: it consists of driving the state from $x(0)=\frac{1}{2}$ to $x\left(\frac{1}{2}\right)=0$ with the first mode, i.e. $u_{1}(t)=1, u_{2}(t)=0$ for $t \in\left[0, \frac{1}{2}[\right.$, and then chattering between the first and second mode with equal proportion so as to keep $x(t)=0$, i.e. $u_{1}(t)=\frac{1}{2}, u_{2}(t)=\frac{1}{2}$ for $\left.\left.t \in\right] \frac{1}{2}, 1\right]$. It follows that the infimum is equal to

$$
p^{*}=\int_{0}^{1 / 2}\left(\frac{1}{2}-t\right)^{2} d t=\frac{1}{24} \approx 4.1667 \cdot 10^{-2} .
$$

Because of chattering, the infimum in problem (1) is not attained by an admissible switching sequence. It is however attained in the convexified problem (3). 


\begin{tabular}{c|c|c|cc}
$d$ & $p_{d}^{*}$ & $N_{d}$ & $y_{1,0}^{d}$ & $y_{2,0}^{d}$ \\
\hline 1 & $-5.9672 \cdot 10^{-9}$ & 18 & 0.74056 & 0.25944 \\
2 & $4.1001 \cdot 10^{-2}$ & 45 & 0.75170 & 0.24830 \\
3 & $4.1649 \cdot 10^{-2}$ & 84 & 0.74632 & 0.25368 \\
4 & $4.1666 \cdot 10^{-2}$ & 135 & 0.74918 & 0.25082 \\
5 & $4.1667 \cdot 10^{-2}$ & 198 & 0.74974 & 0.25026 \\
6 & $4.1667 \cdot 10^{-2}$ & 273 & 0.74990 & 0.25010 \\
7 & $4.1667 \cdot 10^{-2}$ & 360 & 0.74996 & 0.25004
\end{tabular}

Table 1: Lower bounds $p_{d}^{*}$ on the optimal value $p^{*}$ obtained by solving LMI relaxations of increasing orders $d ; N_{d}$ is the number of variables in the LMI problem; $y_{k, 0}^{d}$ is the approximate time spent on each mode $k=1,2$.

The optimal moments can be obtained analytically

$$
\begin{gathered}
y_{1, \alpha}=\int_{0}^{\frac{1}{2}} t^{\alpha} d t+\frac{1}{2} \int_{\frac{1}{2}}^{1} t^{\alpha} d t=\frac{2+2^{-\alpha}}{4+4 \alpha}, \\
y_{2, \alpha}=\frac{1}{2} \int_{\frac{1}{2}}^{1} t^{\alpha} d t=\frac{2-2^{-\alpha}}{4+4 \alpha}
\end{gathered}
$$

and they can be compared with the following moment vectors obtained numerically at the 7th LMI relaxation:

$$
\begin{aligned}
& y_{1}^{7}=\left[\begin{array}{llllll}
0.74996 & 0.31246 & 0.18746 & 0.13277 & 0.10308 \cdots
\end{array}\right] \\
& y_{1}=\left[\begin{array}{lllll}
0.75000 & 0.31250 & 0.18750 & 0.13281 & 0.10313 \cdots
\end{array}\right] \\
& y_{2}^{7}=\left[\begin{array}{lllll}
0.25004 & 0.18754 & 0.14588 & 0.11723 & 0.096919 \cdots
\end{array}\right] \\
& y_{2}=\left[\begin{array}{lllll}
0.25000 & 0.18750 & 0.14583 & 0.11719 & 0.096875 \cdots
\end{array}\right]
\end{aligned}
$$

We observe that the approximate moments $y_{k}^{7}$ closely match the optimal moments $y_{k}$, so that the approximate control law $u_{k}$ extracted from $y_{k}^{7}$ will be almost optimal.

\subsection{Second example}

We revisit the double integrator example with state constraint studied in [18], formulated as the following optimal switching problem:

$$
\begin{aligned}
p^{*}=\inf & T \\
\text { s.t. } \quad & \dot{x}(t)=f_{\sigma(t)}(x(t)) \\
& x(0)=[1,1], \quad x(T)=[0,0] \\
& x_{2}(t) \geq-1, \quad \forall t \in[0, T]
\end{aligned}
$$

where the infimum is w.r.t. to a switching sequence $\sigma:[0, T] \mapsto\{1,2\}$ with free terminal time $T \geq 0$ and affine dynamics

$$
f_{1}:=\left[\begin{array}{c}
x_{2} \\
-1
\end{array}\right], \quad f_{2}:=\left[\begin{array}{c}
x_{2} \\
1
\end{array}\right]
$$


We know from [18] that the optimal sequence consists of starting with mode 1, i.e. $u_{1}(t)=$ $1, u_{2}(t)=0$ for $t \in[0,2]$, then chattering with equal proportion between mode 1 and 2 , i.e. $u_{1}(t)=u_{2}(t)=\frac{1}{2}$ for $t \in\left[2, \frac{5}{2}\right]$ and then eventually driving the state to the origin with mode 2, i.e. $u_{1}(t)=0, u_{2}(t)=1$ for $t \in\left[\frac{5}{2}, \frac{7}{2}\right]$. Here too the infimum $p^{*}=\frac{7}{2}$ is not attained for problem (1), whereas it is attained with the above controls for problem (3).

In Table 1 we report the lower bounds $p_{d}^{*}$ on the optimal value $p^{*}$ obtained by solving LMI relaxations of increasing orders $d$, rounded to 5 significant digits. We also indicate the number of variables (i.e. total number of moments) of each LMI problem, as well as the zeroth order moment of each occupation measure (recall that these are approximations of the time spent on each mode). We observe that the values of the lower bounds and the masses stabilize quickly to the optimal values $p^{*}=\frac{7}{2}, y_{1,0}=\frac{5}{2}, y_{2,0}=\frac{5}{4}$.

\begin{tabular}{c|c|c|cc}
$d$ & $p_{d}^{*}$ & $N_{d}$ & $y_{1,0}^{d}$ & $y_{2,0}^{d}$ \\
\hline 1 & 2.5000 & 30 & 1.7500 & 0.75000 \\
2 & 3.2015 & 105 & 2.1008 & 1.1008 \\
3 & 3.4876 & 252 & 2.2438 & 1.2438 \\
4 & 3.4967 & 495 & 2.2484 & 1.2484 \\
5 & 3.4988 & 858 & 2.2494 & 1.2494 \\
6 & 3.4993 & 1365 & 2.2496 & 1.2497 \\
7 & 3.4996 & 2040 & 2.2498 & 1.2498
\end{tabular}

Table 2: Lower bounds $p_{d}^{*}$ on the optimal value $p^{*}$ obtained by solving LMI relaxations of increasing orders $d ; N_{d}$ is the number of variables in the LMI problem; $y_{k, 0}^{d}$ is the approximate time spent on each mode $k=1,2$.

The optimal switching sequence, which corresponds here to control measures $\omega_{k}(d t)$ with piecewise constant densities, is obtained numerically as explained in Section 5 , by considering the moments of the (weak) derivative of control measures.

\subsection{Third example}

Consider the optimal control problem (1):

$$
\begin{aligned}
p^{*}=\inf & \int_{0}^{\infty}\|x(t)\|_{2}^{2} d t \\
\text { s.t. } & \dot{x}(t)=A_{\sigma(t)} x(t) \\
& x(0)=[0,-1]
\end{aligned}
$$

where the infimum is w.r.t. to a switching sequence $\sigma:[0, \infty) \mapsto\{1,2\}$ and

$$
A_{1}:=\left[\begin{array}{cc}
-1 & 2 \\
1 & -3
\end{array}\right], \quad A_{2}:=\left[\begin{array}{cc}
-2 & -2 \\
1 & -1
\end{array}\right] \text {. }
$$

Since our framework cannot directly accomodate infinite-horizon problems, we introduce a terminal condition $\|x(T)\|_{2}^{2} \leq 10^{-6}$ so that terminal time $T$ is finite. It means that the switching sequence should drive the state in a small ball around the origin.

In Table 3 we report the lower bounds $p_{d}^{*}$ on the optimal value $p^{*}$ obtained by solving LMI relaxations of increasing orders $d$, rounded to 5 significant digits. We also indicate the 


\begin{tabular}{c|c|c|cc}
$d$ & $p_{d}^{*}$ & $N_{d}$ & $y_{1,0}^{d}$ & $y_{2,0}^{d}$ \\
\hline 1 & 0.24294 & 30 & 1.4252 & 1.4489 \\
2 & 0.24340 & 105 & 2.0639 & 1.9237 \\
3 & 0.24347 & 252 & 1.9639 & 1.8922 \\
4 & 0.24347 & 495 & 1.9537 & 1.8904 \\
5 & 0.24347 & 858 & 1.9572 & 1.8872 \\
6 & 0.24347 & 1365 & 1.9677 & 1.8940 \\
7 & 0.24347 & 2040 & 1.9669 & 1.8928
\end{tabular}

Table 3: Lower bounds $p_{d}^{*}$ on the optimal value $p^{*}$ obtained by solving LMI relaxations of increasing orders $d ; N_{d}$ is the number of variables in the LMI problem; $y_{k, 0}^{d}$ is the approximate time spent on each mode $k=1,2$.

number of variables (i.e. total number of moments) of each LMI problem, as well as the zeroth order moment of each occupation measure (recall that these are approximations of the time spent on each mode). We observe that the values of the lower bounds stabilize quickly.

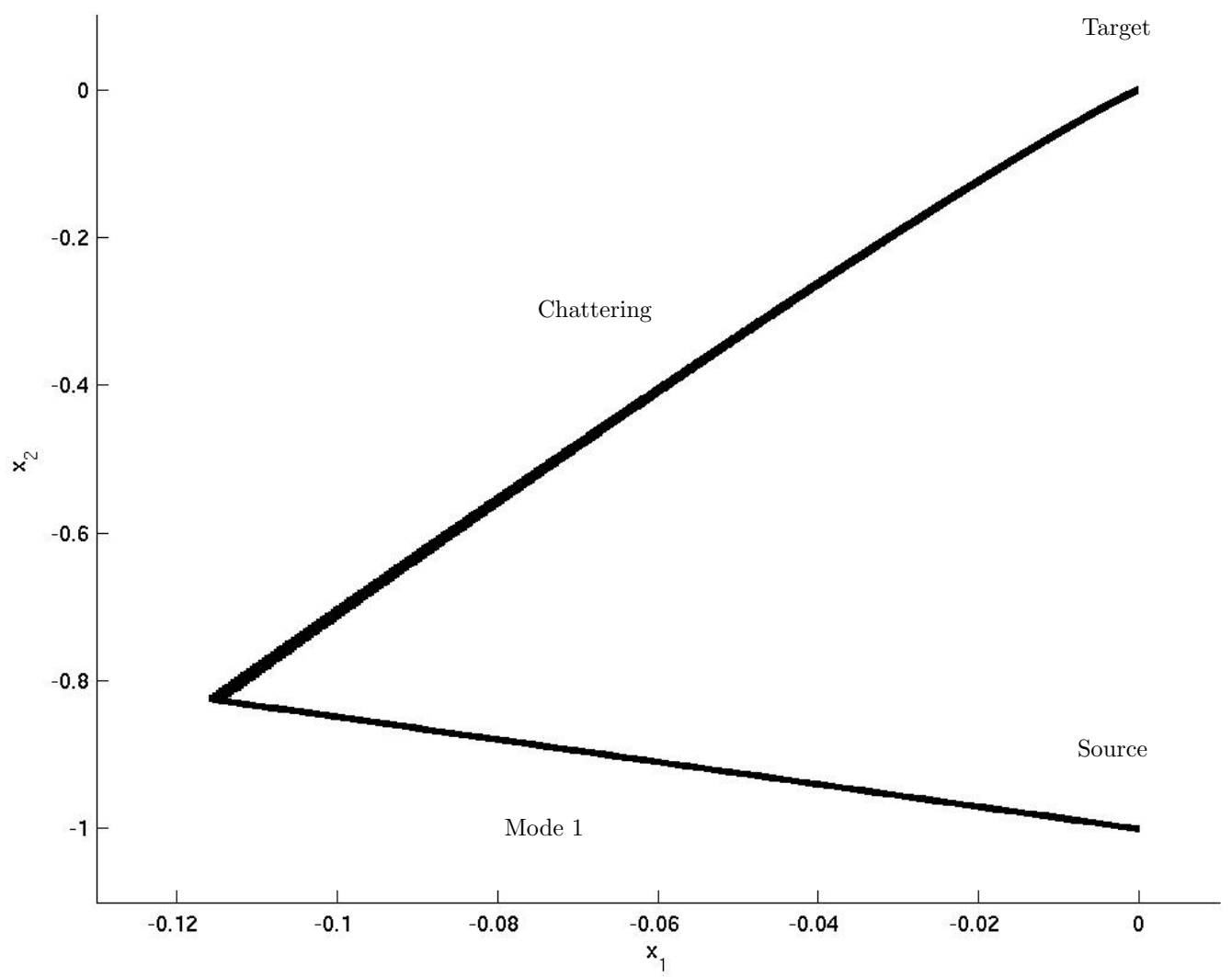

Figure 1: Suboptimal trajectory starting at source point $x=(-1,0)$ with mode 1 , then chattering between modes 1 and 2 to reach the target, a neighborhood of the origin.

In Figure 1 we plot an almost optimal trajectory inferred from the moments of the occu- 
pation measure, for an LMI relaxation of order $d=8$. The trajectory consists in starting from $x=(-1,0)$ with mode 1 during 0.065 time units, and then chattering between mode 1 and mode 2 with respective proportions 49.3/50.7 until $x$ reaches the neighborhood of the origin $\|x(T)\|_{2}^{2} \leq 10^{-6}$ for $T=3.84$. This trajectory is slightly suboptimal, as it yields a cost of 0.24351 , slightly bigger than the guaranteed lower bound of 0.24347 on the best achievable cost obtained by the LMI relxation. It follows that this trajectory is very close to optimality. For comparison with available suboptimal solutions, using [13, Theorem 1], the so-called min switching strategy yields with piecewise quadratic Lyapunov functions a suboptimal trajectory with a cost of 0.24948 .

\section{Conclusion}

In this paper we address the problem of designing an optimal switching sequence for a hybrid system with polynomial Lagrangian (objective function) and polynomial vector fields (dynamics). With the help of occupation measures, we relax the problem from (control) functions with values in $\{0,1\}$ to (control) measures which are absolutely continuous w.r.t. time and summing up to one. This allows for a convex linear programming (LP) formulation of the optimal control problem that can be solved numerically which a classical hierarchy of finite-dimensional convex linear matrix inequality (LMI) relaxations.

We can think of two simple extensions of our approach:

- Open-loop versus closed-loop. In problem (1) the control signal is the switching sequence $\sigma(t)$ which is a function of time: this is an open-loop control, similarly to what was proposed in [7] for impulsive control design. In addition, constrain the switching sequence to be an explicit or implicit function of the state, i.e. $\sigma(x(t))$, a closed-loop control signal. In this case, each occupation measure will be explicitly depending on time, state and control, and it will disintegrate as $\mu(d t, d x, d u)=$ $\xi(d t \mid t, u) \omega(d u \mid t) d t$, and we should follow the framework described originally in [18].

- Switching and impulsive control. We may also combine switching control and impulsive control if we extend the system dynamics (5) to

$$
d x(t)=\sum_{k=1}^{m} f_{k}(x(t)) \omega_{k}(d t)+\sum_{j=1}^{p} g_{j}(t) \tau_{j}(d t)
$$

where $g_{j}$ are given continuous vector functions of time and $\tau_{j}$ are signed measures to be found, jointly with the switching measures $\omega_{k}$. Whereas switching control measures $\omega_{k}$ are restricted by (6) to be absolutely continuous w.r.t. the Lebesgue measure of time, impulsive control measure $\tau_{j}$ can concentrate in time. For example, for a dynamical system $d x(t)=g(t) \tau(d t)$, a Dirac measure $\tau(d t)=\delta_{s}$ enforces at time $t=s$ a state jump $x^{+}(s)=x^{-}(s)+g(s)$. In this case, to avoid trivial solutions, the objective function should penalize the total variation of the impulsive control measures, see [7]. 
- Removing the states in the occupation measures. In the case that all dynamics $f_{k}(t, x), k=1, \ldots, m$ are affine in $x$, we can numerically integrate the state trajectory and approximate the arcs by polynomials of time. It follows that the occupation measures $\mu_{k}(d t, d x)$, once integrated, do not depend on $x$ anymore. They depend on time $t$ only. We can then use finite-dimensional LMI conditions which are necessary and sufficient for a vector to contain the moments of a univariate measure, there is no need to construct a hierarchy of LMI relaxations. There is however still a hierarchy of LMI problems to be solved, now indexed by the degree of the polynomial approximation of the arcs of the state trajectory. To cope with high degree univariate polynomials, alternative bases than monomials are recommended (e.g. Chebyshev polynomials), see [8] for more details.

\section{Acknowledgments}

This work benefited from discussions with Milan Korda, Jean-Bernard Lasserre and Luca Zaccarian.

\section{References}

[1] D. Batenkov. Complete algebraic reconstruction of piecewise-smooth functions from Fourier data. arXiv:1211.0680, Nov. 2012.

[2] S. C. Bengea, R. A. DeCarlo. Optimal control of switching systems. Automatica, 41:11-27, 2005.

[3] M. S. Branicky, V. S. Borkar, S. K. Mitter. A unified framework for hybrid control: model and optimal control theory. IEEE Trans. Autom. Control, 43:31-45, 1998.

[4] A. Bressan, B. Piccoli. Introduction to the mathematical theory of control. Amer. Inst. Math. Sci., Springfield, MO, 2007.

[5] E. J. Candès, C. Fernandez-Granda. Towards a mathematical theory of superresolution. arXiv:1203.5871, Mar. 2012.

[6] C. Cassandras, D. L. Pepyne, Y. Wardi. Optimal control of a class of hybrid systems. IEEE Trans. Autom. Control, 46:398-415, 2001.

[7] M. Claeys, D. Arzelier, D. Henrion, J. B. Lasserre. Measures and LMI for impulsive optimal control with applications to space rendezvous problems. Proc. Amer. Control Conf., Montréal, Canada, 2012.

[8] M. Claeys, D. Arzelier, D. Henrion, J. B. Lasserre. Moment LMI approach to LTV impulsive control. Work in progress, Feb. 2013.

[9] G. S. Deaecto, J. C. Geromel, J. Daafouz. Dynamic output feedback $\mathrm{H}_{\infty}$ control of switched linear systems. Automatica, 47:1713-1720, 2011. 
[10] R. A. DeCarlo, M. S. Branicky, S. Pettersson, B. Lennartson. Perspectives and results on the stability and stabilizability of hybrid systems. Proc. of the IEEE, $88: 1069-1082,2000$.

[11] Y. de Castro, F. Gamboa. Exact reconstruction using Beurling minimal extrapolation. J. Math. Anal. Appl. 395(1):336-354, 2012.

[12] H. O. Fattorini. Infinite dimensional optimization and control theory. Cambridge Univ. Press, Cambridge, UK, 1999.

[13] J. C. Geromel, P. Colaneri, P. Bolzern. Dynamic output feedback control of switched linear systems. IEEE Trans. Autom. Control, 53:720-733, 2008.

[14] J.C. Geromel, G. Deaecto, J. Daafouz. Suboptimal switching control consistency analysis for switched linear systems. To appear in IEEE Trans. Autom. Control, 2013.

[15] S. Hedlund, A. Rantzer. Optimal control of hybrid systems. Proc. IEEE Conf. Decision and Control, Pheonix, Arizona, 1999.

[16] D. Henrion, M. Korda. Convex computation of the region of attraction of polynomial control systems. arXiv:1208.1751, Aug. 2012.

[17] D. Henrion, J. B. Lasserre, M. Mevissen. Mean squared error minimization for inverse moment problems. arXiv:1208.6398, Aug. 2012.

[18] J. B. Lasserre, D. Henrion, C. Prieur, E. Trélat. Nonlinear optimal control via occupation measures and LMI relaxations. SIAM J. Control Opt., 47:1643-1666, 2008 .

[19] J. B. Lasserre. Moments, positive polynomials and their applications. Imperial College Press, London, UK, 2009.

[20] D. Liberzon, A. S. Morse. Basic problems in stability and design of switched systems. IEEE Control Systems Mag. 19:59-70, 1999.

[21] D. Liberzon. Switching in systems and control. Birkhaeuser, Basel, 2003.

[22] H. Lin, P. J. Antsaklis. Stability and stabilizability of switched linear systems: A survey of recent results. IEEE Trans. Autom. Control, 54:308-322, 2009.

[23] B. Piccoli. Hybrid systems and optimal control. Proc. IEEE Conf. Decision and Control, Tampa, Florida, 1998.

[24] P. Riedinger, C. Zanne, F. Kratz. Time optimal control of hybrid systems. Proc. Amer. Control Conf., San Diego, California, 1999.

[25] P. Riedinger, C. Iung, F. Kratz. An optimal control approach for hybrid systems. Europ. J. Control, 9:449-458, 2003.

[26] C. Seatzu, D. Corona, A. Giua, A. Bemporad. Optimal control of continuous-time switched affine systems. IEEE Trans. Autom. Control, 51:726-741, 2006. 
[27] H.J. Sussmann. A maximum principle for hybrid optimization. Proc. IEEE Conf. Decision and Control, Phoenix, Arziona, 1999.

[28] M. S. Shaikh, P. E. Caines. On the hybrid optimal control problem: theory and algorithms. IEEE Trans. Autom. Control, 52:1587-1603, 2007.

[29] R. Shorten, F. Wirth, O. Mason, K. Wulff, C. King, Stability criteria for switched and hybrid systems. SIAM Review, 49:545-592, 2007.

[30] Z. Sun, S. S. Ge. Switched linear systems: control and design. Springer, London, 2005 .

[31] X. Xu, P. J. Antsaklis. Results and perspectives on computational methods for optimal control of switched systems. In: O. Maler, A. Pnueli, A. (eds.), HSCC 2003, Lecture Notes Comput. Sci., 2623:540-555, Springer, Heidelberg, 2003. 